\title{
Obstructive Sleep Apnea and Chronic Lung Disease
}

\author{
Woo Ho Ban' and Sang Haak Lee² \\ 'Division of Pulmonary, Allergy and Critical Care Medicine, Department of Internal Medicine, Seoul St. Mary's Hospital, \\ College of Medicine, The Catholic University of Korea, Seoul, Korea \\ 2Division of Pulmonary, Critical Care and Sleep Medicine, Department of Internal Medicine, St. Paul's Hospital, \\ College of Medicine, The Catholic University of Korea, Seoul, Korea
}

\begin{abstract}
Idiopathic pulmonary fibrosis (IPF) and chronic obstructive pulmonary disease (COPD) are chronic disabling lung diseases that progress slowly in the elderly and are characterized by remodeling of the lung interstitium or bronchoalveolar structure. Patients with IPF or COPD have various comorbidities such as pulmonary arterial hypertension, ischemic heart disease, sleep-disordered breathing, and even cancer. Chronic hypoxia is one of the most important mechanisms in these patients and promotes changes in the lung parenchyma and vascular structure via systemic inflammation and endothelial dysfunction. Research on sleep disturbance in IPF and COPD has been vigorously conducted, and there have been reports in recent years that have suggested that obstructive sleep apnea (OSA) is closely related to disease progression and poor clinical outcomes in these two diseases. Intermittent hypoxia $(\mathrm{IH})$, a key mechanism of OSA, induces further systemic inflammation and sympathetic overactivity, which may aggravate disease progression in IPF and COPD, which characteristically predispose to chronic hypoxia. Epidemiologic and experimental evidence has shown that OSA and IH could be promising targets to prevent the progression of IPF and COPD. This review provides a possible pathological mechanism of OSA and $\mathrm{IH}$ and suggests their promising role as emerging targets for disease control in IPF and COPD.
\end{abstract}

Key Words: Sleep apnea; Obstructive; Pulmonary disease; Chronic obstructive; Idiopathic pulmonary fibrosis

Received: January 4, 2019 Revised: January 15, 2019 Accepted: January 28, 2019

Corresponding author: Sang Haak Lee, MD, PhD, Division of Pulmonary, Critical Care and Sleep Medicine, Department of Internal Medicine, St. Paul's Hospital, College of Medicine, The Catholic University of Korea, 180 Wangsan-ro, Dongdaemun-gu, Seoul 62056, Korea.

Tel: 82-2-961-4500, Fax: 82-2-968-7250, E-mail: mdlee@catholic.ac.kr

@ This is an Open Access article distributed under the terms of the Creative Commons Attribution Non-Commercial License (https://creativecommons.org/licenses/by$\mathrm{nc} / 4.0)$ which permits unrestricted non-commercial use, distribution, and reproduction in any medium, provided the original work is properly cited.

\section{INTRODUCTION}

Idiopathic pulmonary fibrosis (IPF) and chronic obstructive pulmonary disease (COPD) are typical chronic lung diseases that progress slowly in the elderly. Recent years have seen the development of new anti-fibrotic agents and ongoing development of inhalers, and there have been improvements in the control of both diseases. However, the mortality and clinical outcomes of IPF and COPD remain of concern, and the burden of illness and economic cost to patients and society remain high [1,2]. IPF is characterized primarily by the restriction of pulmonary function due to fibrosis of the interstitial area, while COPD is characterized by the obstruction of pulmonary function due to an emphysematous change in alveolar and bronchiolar inflammation. However, the two diseases have several commonalities. Systemic inflammation and excessive oxidative stress play an important role in the exacerbation and outcome of the two diseases. Moreover, exacerbation events accelerate the vicious cycle of these diseases. In addition, the prevalence rates of ischemic heart disease, pulmonary hypertension, cancer, and sleep disturbance are higher in IPF and COPD than in the general population [3-5]. Among these, obstructive sleep apnea (OSA), in particular, has been highlighted in recent years as one of the most promising targets to prevent disease progression and improve quality of life.

OSA is a sleep-related breathing disorder characterized by repetitive disruption of sleep due to collapse of the upper airway [6]. Intermittent hypoxia (IH) is the most important pathological marker in OSA, which also causes systemic inflammation and excessive oxidative stress. The association of OSA with various chronic and metabolic diseases is being increasingly reported. Re- 
cent advances in the testing and treatment of OSA have elucidated this association in patients with chronic disease. For example, OSA and cardiovascular disease outcomes have been studied extensively, and treatment of OSA to prevent cardiovascular disease is promising. However, there have been relatively few theoretical studies regarding the role of OSA in chronic respiratory diseases. Therefore, we focused on the relationships of IPF and COPD with OSA, in terms of epidemiological and experimental results, and suggest a putative role of OSA as a target for control of IPF and COPD.

\section{EPIDEMIOLOGIC EVIDENCE: OSA AS A DISEASE-AGGRAVATING FACTOR IN IPF AND COPD}

\section{Epidemiologic evidence of the association between OSA} and IPF

Overall, published research has reported a distortion of sleep architecture, including decreased sleep efficiency, increased sleep fragmentation, and sleep-related hypoventilation in patients with IPF, while OSA was also frequently observed $[7,8]$. The reported prevalence of OSA in the literature is 6-91\%, and several reports have suggested its close association with quality of life and prognosis in patients with IPF [9]. After the IPF diagnostic criteria were more clearly defined in 2002 by the American Thoracic Society/European Respiratory Society, studies on sleep disorders in IPF patients began to be conducted in earnest. Since the Cleveland Clinic Sleep Disorders Center reported an OSA prevalence of about $60 \%$ in a retrospective study of 18 IPF patients who underwent polysomnography (PSG), several additional studies have been conducted on sleep-disordered breathing (SDB) in IPF [8]. In most of these studies, the prevalence of OSA was significantly higher in IPF patients than in a healthy population of the same age $[10,11]$. The exact mechanism underlying the high prevalence of OSA in patients with IPF has not yet been established. Reduced lung volume has been considered one of the main causes [12]. In general, upper-airway resistance increases during sleep, while functional residual capacity (FRC) decreases. When seeking to elevate the lung volume beyond the decreased FRC, increased respiratory effort exerted via negative extrathoracic pressure frequently leads to collapse of the upper airway [13]. Therefore, OSA is more common in IPF patients than in the general population. Moreover, OSA is associated with various comorbidities such as pulmonary hypertension, gastroesophageal reflux disease, and diabetes; these comorbidities are important factors driving disease progression [14-16]. Recently, a single-center observational study was conducted in Italy that enrolled 35 IPF patients after PSG. They found that the more severe the OSA and hypoxemia, the higher the rates of mortality and clinical deterioration [17]. Although the small number of study patients was a limitation, there is growing evidence to indicate that OSA induces IPF aggravation and is closely associated with poor outcomes.
Epidemiologic evidence of the association between OSA and COPD

The epidemiologic evidence for an interaction between COPD and OSA is still under debate. Zhao et al. [18] showed that the prevalence of COPD was lower among patients with SDB than among those without SDB, while Soler et al. [19] reported a higher prevalence of OSA in patients with moderate-to-severe COPD. These contrasting findings are due to the nature of COPD, which is usually diagnosed when an irreversible airway obstruction is noted in a pulmonary function test. However, there is a wide spectrum of disease phenotypes associated with such abnormal airway obstruction. The heterogeneity of these diseases, which include an emphysema phenotype, chronic bronchitis phenotype, and elderly COPD, appears to be the reason for the difficulty in clarifying the relationship between COPD and OSA. It has recently been reported that the prevalence of OSA varies according to body mass index (BMI) and COPD type. Patients with COPD, those with dominant emphysema, and those with a low BMI may have a lower likelihood of OSA. On the other hand, patients with a higher BMI, those with a chronic bronchitis phenotype, and those with cor pulmonale are predisposed to OSA [20,21]. There are several pathological mechanisms linking OSA and COPD. Upper-airway inflammation associated with cigarette smoking [22] and myopathy of the upper-airway muscles caused by inhaled corticosteroids [23] may induce OSA in patients with COPD. Moreover, OSA exacerbates COPD by promoting inflammation of the lower respiratory tract [24]. Recently, combined COPD and OSA has been referred to as overlap syndrome, which constitutes a distinct disease entity; such patients show poor clinical characteristics and outcomes compared to COPD-only patients in general. The Spanish Sleep Cohort Study showed that COPD-OSA overlap syndrome patients not undergoing continuous positive airway pressure (CPAP) treatment had increased rates of hospitalization and death due to COPD exacerbation compared to a COPD-only group [25]. There is therefore a growing consensus that certain phenotypes of COPD are responsible for a high proportion of OSA, and that OSA could impact significantly on COPD deterioration and prognosis.

\section{INTERMITTENT HYPOXIA AND CHRONIC HYPOXIA: CRITICAL FEATURES IN IPF AND COPD COMBINED WITH OSA}

\section{Chronic hypoxia in IPF and COPD}

The most important clinical feature of IPF and COPD, and the accepted index of disease progression, is hypoxia. V/Q mismatch and diffusion impairment are the main mechanisms of hypoxia in the two diseases [26,27]. Fibroblast and myofibroblast proliferation and excessive deposition of disorganized collagen and extracellular matrix are the main pathological features of IPF, resulting in distortion of the normal lung alveoli, interstitial spaces, and vasculature structures [28]. In COPD, on the other hand, progres- 
sive airflow limitation due to airway inflammation and emphysematous destruction of the alveolar and pulmonary capillaries are observed during disease progression [27]. These changes gradually induce chronic hypoxia and, in advanced cases, eventually lead to pulmonary vasoconstriction and pulmonary hypertension.

Various mechanisms exist that may explain IPF and COPD deterioration. The most important mechanism is systemic inflammation arising from chronic hypoxia. In IPF, persistent hypoxia activates cytokines such as protein kinase $\mathrm{C}$ and interleukin (IL)1, which stimulate vasoconstriction, fibroblast expression, and leukocyte recruitment [29]. Recruited inflammatory cells release matrix metalloproteinases, transforming growth factor beta, and tumor necrosis factor alpha (TNF- $\alpha$ ), and cause collagen deposition leading to IPF progression [30]. This vicious cycle between chronic hypoxia and fibrosis accelerates IPF progression. Several studies have also reported that endothelial cell dysfunction and remodeling due to chronic hypoxia cause microvascular injury, which is associated with IPF progression [31]. Similarly, chronic hypoxia in COPD patients induces systemic inflammation via increased levels of circulatory cytokines, such as TNF- $\alpha$ and IL-6 [32], and causes excessive oxidative stress [33]. Such systemic inflammation and excessive oxidative stress due to chronic hypoxia cause cardiovascular morbidity, as well as progression of COPD itself.

\section{IH in IPF and COPD combined with OSA}

$\mathrm{IH}$, a condition in which periods of normoxia alternate with hypoxia, is a representative pathognomic mechanism induced by OSA. Patients with OSA experience repetitive episodes of apnea and hypopnea during the sleep cycle, leading to excessive systemic inflammation and overproduction of reactive oxygen species. These critical processes during IH in OSA cause endothelial dam- age and further tissue damage [34]. Various comorbidities of OSA are linked to IH. Metabolic dysfunction and vascular complications in patients with OSA are usually caused by this effect [35] which is also closely related to systemic inflammatory markers such as TNF- $\alpha$ and IL-8, which are the major cytokines associated with disease progression in IPF and COPD [36]. The presence of such overlapping molecules may represent evidence that OSA is closely related to IPF and COPD progression, and that patients with combined OSA have a larger disease burden and increased systemic inflammation compared to those without OSA in IPF or COPD. In addition, as in OSA, IPF and COPD patients show sympathetic overactivity and endothelial dysfunction. Cardiovascular disease and pulmonary hypertension are frequently observed in IPF and COPD patients, as in OSA [9,37]. However, it remains unclear whether $\mathrm{IH}$ or chronic hypoxia promotes more severe systemic inflammation and vascular remodeling. In IPF and COPD patients, who are already predisposed to chronic hypoxemia and sympathetic over-activity, exposure to additional IH increases the duration and frequency of hypoxia and promotes further systemic inflammation, sympathetic over-activity, and vascular remodeling (Figure 1). Obviously, IPF and COPD patients with OSA have poorer clinical outcomes, such as higher levels of cardiovascular disease and decreased survival, compared to those without OSA $[38,39]$.

\section{RECENT EXPERIMENTAL EVIDENCE OF IH AS A POSSIBLE AGGRAVATOR OF IPF AND COPD}

Many studies on the cardiovascular complications of IH and OSA have focused on remodeling of the vasculature. However,

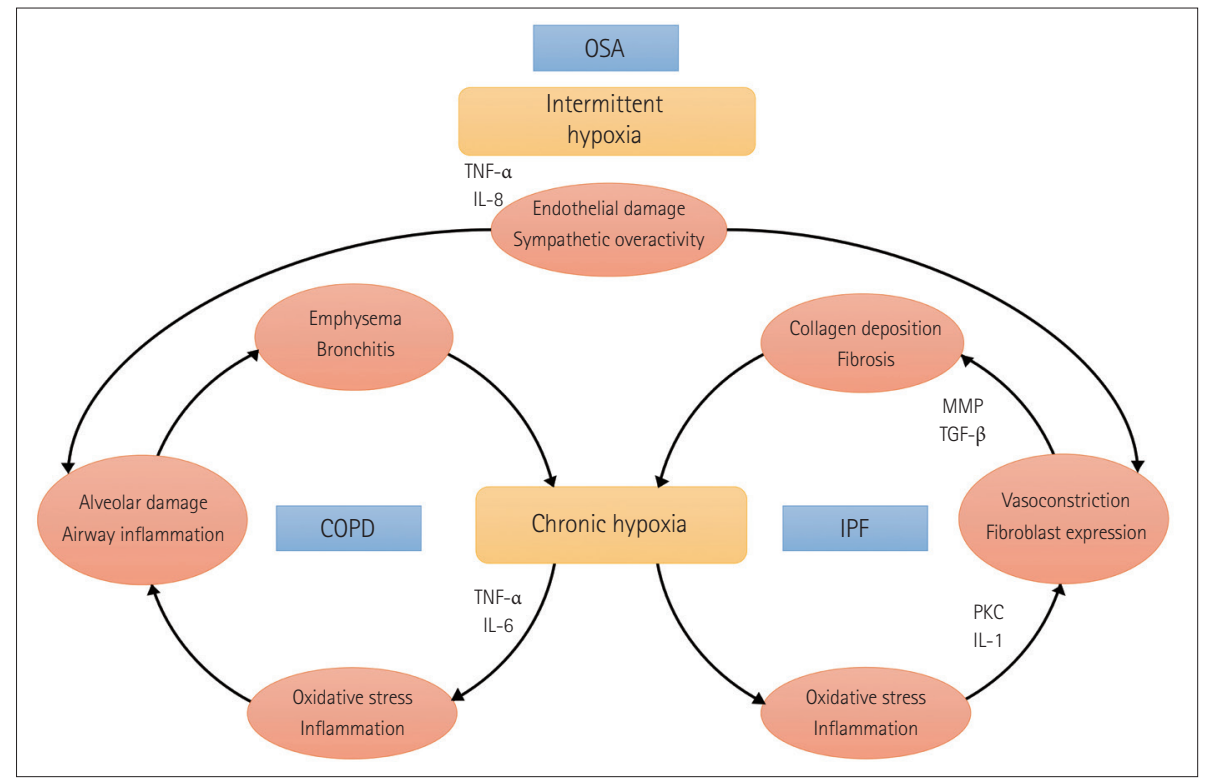

Figure 1. Effect of chronic hypoxia and IH on IPF and COPD progression. Predisposition to chronic hypoxia induces oxidative stress and systemic inflammation in IPF and COPD. Overlap with IH increases the duration and severity of hypoxia, accelerating disease progression in patients with IPF or COPD combined with OSA. IPF: idiopathic pulmonary fibrosis, COPD: chronic obstructive pulmonary disease, OSA: obstructive sleep apnea, TNF-a: tumor necrosis factor alpha, IL-8: interleukin-8, IL-6: interleukin-6, IL-1: interleukin-1, PKC: protein kinase C, MMP: matrix metalloproteinases, TGF- $\beta$ : transforming growth factor beta. 
studies on the effects of IH on the deterioration of pulmonary disease are lacking. Using in vivo experiments, recent studies have shown that IH may also affect the course of lung fibrosis. Gille et al. [40] suggested that chronic IH promoted lung fibrosis and increased mortality in a bleomycin-induced lung-injury mouse model. They demonstrated that exposure to chronic IH worsened bleomycin-induced lung injury via increased neutrophilic pulmonary inflammation, lung cell apoptosis, increased collagen accumulation, and an imbalance between antioxidants and prooxidants. In the area of COPD experimental models, Yang et al. [41] demonstrated that IH promoted more severe systemic inflammation, endothelial inflammation and endothelial damage than seen in controls in an emphysematous rat model. Li et al. [42] reported similar results, in that rats with emphysema and $\mathrm{IH}$ had a higher pro-inflammatory and pro-thrombotic status than controls. Several studies have been conducted on excessive inflammation in rat models of overlap syndrome, although few have been conducted to determine whether $\mathrm{IH}$ initiates or promotes the progression of COPD. Additional experiments are needed to determine whether blocking $\mathrm{IH}$ can serve as a promising therapeutic target for COPD.

\section{OSA: NON-PHARMACOLOGICAL THERAPEUTIC TARGET OF IPF AND COPD}

In recent years, clinical data have emerged showing that OSA and IH may be major targets to prevent disease progression and improve prognosis in IPF and COPD. Kolilekas et al. [38] showed that intermittent sleep oxygen desaturation was closely associated with functional impairment and poor survival outcomes in 31 IPF patients who underwent PSG. They suggested that IH could be a major target for improving the prognosis of IPF. Mermigkis et al. [43] analyzed the effect of CPAP treatment on the prognosis of 55 patients with combined IPF and OSA. Despite study limitations such as a short follow-up duration and small population, the CPAP group that showed good compliance nevertheless had better quality of sleep and survival outcomes compared to the CPAP group that showed poor compliance. Similar results have been obtained in COPD patients. Marin et al. [25] demonstrated that patients with COPD-OSA overlap syndrome treated with longterm CPAP had favorable survival outcomes compared to nontreated patients in a 10-year follow-up study. Machado et al. [44] reported similar results: patients with OSA and COPD receiving CPAP showed higher survival in their prospective cohort study in Brazil.

In light of the above, active surveillance of OSA and IH via PSG is necessary in IPF and COPD patients. In addition, active management using CPAP is required to delay disease progression in patients with IPF or COPD combined with OSA. This will be a major clinical non-pharmacologic target in IPF and COPD patients in the future, as will long-term oxygen therapy.

\section{CONCLUSION AND FUTURE RESEARCH PERSPECTIVES}

Chronic hypoxia promotes systemic inflammation and pulmonary vascular damage in IPF and COPD. In addition, the prevalence of OSA is significantly higher in these two diseases than in the general population. If IH, which is a key mechanism of OSA, overlaps with IPF and COPD, it induces greater oxidative stress and systemic inflammation, and accelerates lung structural damage and endothelial remodeling in IPF and COPD. Recent clinical and experimental data have shown that IH is closely associated with disease progression and a worse outcome in IPF and COPD. Additionally, treatment of $\mathrm{IH}$ could attenuate disease progression and improve clinical outcomes. Therefore, clinicians need to diagnose OSA early in IPF and COPD through active surveillance via PSG, and intervene with CPAP to improve the prognosis of IPF and COPD. Moreover, in vivo and in vitro experiments focusing on identifying distinct mechanisms linking $\mathrm{IH}$ with the initiation or progression of the two diseases are needed to establish clearer evidence. In addition, a large-scale prospective clinical study to clarify the clinical impact of treating OSA in patients with IPF and COPD should be performed in the future.

\section{Conflicts of Interest}

The authors have no potential conflicts of interest to disclose.

\section{Author Contributions}

Conceptualization: Sang Haak Lee. Data curation: Woo Ho Ban. Formal analysis: Woo Ho Ban, Sang Haak Lee. Investigation: Woo Ho Ban. Methodology: Woo Ho Ban, Sang Haak Lee. Project administration: Sang Haak Lee. Resources: Woo Ho Ban. Supervision: Sang Haak Lee. Visualization: Woo Ho Ban, Sang Haak Lee. Writing_original draft: Woo Ho Ban. Writing_review \& editing: Sang Haak Lee.

\section{ORCID iDs}

Sang Haak Lee (1)

https://orcid.org/0000-0001-6259-7656

Woo Ho Ban (10)

https://orcid.org/0000-0002-7115-0490

\section{REFERENCES}

1. Lee AS, Mira-Avendano I, Ryu JH, Daniels CE. The burden of idiopathic pulmonary fibrosis: an unmet public health need. Respir Med 2014;108: 955-967.

2. Thun MJ, Carter BD, Feskanich D, Freedman ND, Prentice R, Lopez AD, et al. 50-year trends in smoking-related mortality in the United States. N Engl J Med 2013;368:351-364.

3. Raghu G, Amatto VC, Behr J, Stowasser S. Comorbidities in idiopathic pulmonary fibrosis patients: a systematic literature review. Eur Respir J 2015; 46:1113-1130.

4. Sin DD, Man SF. Why are patients with chronic obstructive pulmonary disease at increased risk of cardiovascular diseases? The potential role of systemic inflammation in chronic obstructive pulmonary disease. Circulation 2003;107:1514-1519.

5. Sin DD, Anthonisen NR, Soriano JB, Agusti AG. Mortality in COPD: role of 
comorbidities. Eur Respir J 2006;28:1245-1257.

6. Park JG, Ramar K, Olson EJ. Updates on definition, consequences, and management of obstructive sleep apnea. Mayo Clin Proc 2011;86:549-554; quiz 554-555.

7. Mermigkis C, Stagaki E, Amfilochiou A, Polychronopoulos V, Korkonikitas P, Mermigkis D, et al. Sleep quality and associated daytime consequences in patients with idiopathic pulmonary fibrosis. Med Princ Pract 2009;18:10-15.

8. Mermigkis C, Chapman J, Golish J, Mermigkis D, Budur K, Kopanakis A, et al. Sleep-related breathing disorders in patients with idiopathic pulmonary fibrosis. Lung 2007;185:173-178.

9. Schiza S, Mermigkis C, Margaritopoulos GA, Daniil Z, Harari S, Poletti V, et al. Idiopathic pulmonary fibrosis and sleep disorders: no longer strangers in the night. Eur Respir Rev 2015;24:327-339.

10. Lancaster LH, Mason WR, Parnell JA, Rice TW, Loyd JE, Milstone AP, et al. Obstructive sleep apnea is common in idiopathic pulmonary fibrosis. Chest 2009;136:772-778.

11. Pihtili A, Bingol Z, Kiyan E, Cuhadaroglu C, Issever H, Gulbaran Z. Obstructive sleep apnea is common in patients with interstitial lung disease. Sleep Breath 2013;17:1281-1288.

12. Heinzer RC, Stanchina ML, Malhotra A, Fogel RB, Patel SR, Jordan AS, et al. Lung volume and continuous positive airway pressure requirements in obstructive sleep apnea. Am J Respir Crit Care Med 2005;172:114-117.

13. Hudgel DW, Devadatta P. Decrease in functional residual capacity during sleep in normal humans. J Appl Physiol Respir Environ Exerc Physiol 1984; 57:1319-1322.

14. Pitsiou G, Papakosta D, Bouros D. Pulmonary hypertension in idiopathic pulmonary fibrosis: a review. Respiration 2011;82:294-304.

15. Fahim A, Crooks M, Hart SP. Gastroesophageal reflux and idiopathic pulmonary fibrosis: a review. Pulm Med 2011;2011:634613.

16. Enomoto T, Usuki J, Azuma A, Nakagawa T, Kudoh S. Diabetes mellitus may increase risk for idiopathic pulmonary fibrosis. Chest 2003;123:20072011.

17. Bosi M, Milioli G, Fanfulla F, Tomassetti S, Ryu JH, Parrino L, et al. OSA and prolonged oxygen desaturation during sleep are strong predictors of poor outcome in IPF. Lung 2017;195:643-651.

18. Zhao YY, Blackwell T, Ensrud KE, Stone KL, Omachi TA, Redline S. Sleep apnea and obstructive airway disease in older men: outcomes of sleep disorders in older men study. Sleep 2016;39:1343-1351.

19. Soler X, Gaio E, Powell FL, Ramsdell JW, Loredo JS, Malhotra A, et al. High prevalence of obstructive sleep apnea in patients with moderate to severe chronic obstructive pulmonary disease. Ann Am Thorac Soc 2015;12:12191225.

20. Krachman SL, Tiwari R, Vega ME, Yu D, Soler X, Jaffe F, et al. Effect of emphysema severity on the apnea-hypopnea index in smokers with obstructive sleep apnea. Ann Am Thorac Soc 2016;13:1129-1135.

21. White LH, Bradley TD. Role of nocturnal rostral fluid shift in the pathogenesis of obstructive and central sleep apnoea. J Physiol 2013;591:1179-1193.

22. Renner B, Mueller CA, Shephard A. Environmental and non-infectious factors in the aetiology of pharyngitis (sore throat). Inflamm Res 2012;61:10411052 .

23. Teodorescu M, Consens FB, Bria WF, Coffey MJ, McMorris MS, Weatherwax KJ, et al. Predictors of habitual snoring and obstructive sleep apnea risk in patients with asthma. Chest 2009;135:1125-1132.

24. Wang Y, Hu K, Liu K, Li Z, Yang J, Dong Y, et al. Obstructive sleep apnea exacerbates airway inflammation in patients with chronic obstructive pulmonary disease. Sleep Med 2015;16:1123-1130.
25. Marin JM, Soriano JB, Carrizo SJ, Boldova A, Celli BR. Outcomes in patients with chronic obstructive pulmonary disease and obstructive sleep apnea: the overlap syndrome. Am J Respir Crit Care Med 2010;182:325-331.

26. Agustí AG, Roca J, Gea J, Wagner PD, Xaubet A, Rodriguez-Roisin R. Mechanisms of gas-exchange impairment in idiopathic pulmonary fibrosis. Am Rev Respir Dis 1991;143:219-225.

27. Kent BD, Mitchell PD, McNicholas WT. Hypoxemia in patients with COPD: cause, effects, and disease progression. Int J Chron Obstruct Pulmon Dis 2011;6:199-208.

28. Barratt SL, Creamer A, Hayton C, Chaudhuri N. Idiopathic pulmonary fibrosis (IPF): an overview. J Clin Med 2018;7:201.

29. Strieter RM. Mechanisms of pulmonary fibrosis: conference summary. Chest 2001;120(1 Suppl):77S-85S.

30. Winkler MK, Fowlkes JL. Metalloproteinase and growth factor interactions: do they play a role in pulmonary fibrosis? Am J Physiol Lung Cell Mol Physiol 2002;283:L1-L11.

31. Magro CM, Waldman WJ, Knight DA, Allen JN, Nadasdy T, Frambach GE, et al. Idiopathic pulmonary fibrosis related to endothelial injury and antiendothelial cell antibodies. Hum Immunol 2006;67:284-297.

32. Gan WQ, Man SF, Senthilselvan A, Sin DD. Association between chronic obstructive pulmonary disease and systemic inflammation: a systematic review and a meta-analysis. Thorax 2004;59:574-580.

33. MacNee W. Oxidants/antioxidants and COPD. Chest 2000;117(5 Suppl 1): 303S-317S

34. Prabhakar NR, Kumar GK. Oxidative stress in the systemic and cellular responses to intermittent hypoxia. Biol Chem 2004;385:217-221.

35. Dewan NA, Nieto FJ, Somers VK. Intermittent hypoxemia and OSA: implications for comorbidities. Chest 2015;147:266-274.

36. Nadeem R, Molnar J, Madbouly EM, Nida M, Aggarwal S, Sajid H, et al. Serum inflammatory markers in obstructive sleep apnea: a meta-analysis. J Clin Sleep Med 2013;9:1003-1012.

37. Taranto-Montemurro L, Messineo L, Perger E, Salameh M, Pini L, Corda L, et al. Cardiac sympathetic hyperactivity in patients with chronic obstructive pulmonary disease and obstructive sleep apnea. COPD 2016;13:706-711.

38. Kolilekas L, Manali E, Vlami KA, Lyberopoulos P, Triantafillidou C, Kagouridis $\mathrm{K}$, et al. Sleep oxygen desaturation predicts survival in idiopathic pulmonary fibrosis. J Clin Sleep Med 2013;9:593-601.

39. Chaouat A, Weitzenblum E, Krieger J, Ifoundza T, Oswald M, Kessler R. Association of chronic obstructive pulmonary disease and sleep apnea syndrome. Am J Respir Crit Care Med 1995;151:82-86.

40. Gille T, Didier M, Rotenberg C, Delbrel E, Marchant D, Sutton A, et al. Intermittent hypoxia increases the severity of bleomycin-induced lung injury in mice. Oxid Med Cell Longev 2018;2018:1240192.

41. Yang QC, Sun X, Wang YM, Wu Q, Feng J, Chen BY. Systematic and endothelial inflammation and endothelial progenitor cell levels in emphysematous rats exposed to intermittent hypoxia. Respir Care 2015;60:279-289.

42. Li C, Yang X, Feng J, Lei P, Wang Y. Proinflammatory and prothrombotic status in emphysematous rats exposed to intermittent hypoxia. Int J Clin Exp Pathol 2015;8:374-383.

43. Mermigkis C, Bouloukaki I, Antoniou K, Papadogiannis G, Giannarakis I, Varouchakis G, et al. Obstructive sleep apnea should be treated in patients with idiopathic pulmonary fibrosis. Sleep Breath 2015;19:385-391.

44. Machado MC, Vollmer WM, Togeiro SM, Bilderback AL, Oliveira MV, Leitão FS, et al. CPAP and survival in moderate-to-severe obstructive sleep apnoea syndrome and hypoxaemic COPD. Eur Respir J 2010;35:132-137. 